Jurnal Keperawatan Padjadjaran

ISSN 2338-5324 (print)

ISSN 2442-7276 (online)

Online di http://jkp.fkep.unpad.ac.id

DOI : $10.24198 / \mathrm{jkp}$

\title{
An Exploration the Risk of Cardiovascular Disease in HIV-Positive Persons in Indonesia using Heart Rate Variability
}

\author{
Linlin Lindayani ${ }^{1}$, Irma Darmawati ${ }^{2}$, Heni Purnama ${ }^{3}$, Pujowati ${ }^{4}$, Taryudi ${ }^{5}$ \\ ${ }^{1,2,3}$ STIKep PPNI West Java, Bandung, Indonesia \\ ${ }^{4}$ RSUP Hasan Sadikin, Bandung, Indonesia \\ ${ }^{5}$ Faculty of Electrical Engineering, Universitas Negeri Jakarta, Jakarta Indonesia \\ Corresponding email: linlinlindayani@gmail.com
}

Submitted: 07-07-2018 Accepted: 16-09-2019 Published: 05-12-2019

\begin{abstract}
Cardiovascular disease is the highest cause of death in HIV patients compared to the general population. The number of HIV patients suffering from cardiovascular disease is almost twice as high as patients who are not HIV-positive. The purpose of this study was to identify the risk of cardivascular disease in patients with HIV using ECG short term. This study was used a descriptive comparative to patients with HIV and healthy people as controls in West Java. The inclusion criteria are patients with HIV over the age of 30 years. The exclusion criteria were people with HIV diagnosed with heart disease or being treated for the heart disease. While the inclusion criteria for healthy people as controls are over 30 years of age, do not suffer from cardivascular disease or under treatment of cardiovascular disease. The measurement of heart rate variability is carried out in a supine position in a quiet temperature-controlled room (25-270 C), a 5-minute electrocardiograph (ECG) is recorded using lead II. Differences of heart rate variability indicator were measure using man-whitney test. A total of 20 patients with HIV and 20 healthy people recruited using convinience sampling. The majority of people with HIV were male and aged range between 27 to 51 years old. The results of heart rate variability based on time domain analysis showed that the means normal to normal (NN) was significantly lower in HIV patients compared to controls (978 vs ?? vs $902 \mathrm{~ms} ; \mathrm{p}<0.05)$. No differences were found between groups regarding Standard deviation of NN (SDNN), Square root of the mean squared difference of successive NN-intervals (RMSSD) and Percent of differences between adjacent NN intervals greater than 50 $\mathrm{ms}$ (pNN50). This study presence of autonomic dysfunction as showed in heart rate variability indicator in a group of HIV compared to the healthy group. Eearly identification of the risk of CVD is important and may inform the implementation of preventive measure by identification of high-risk people who may be candidate for intervention.
\end{abstract}

Keywords: Cardivascular disease, HIV, heart rate variability, HRV, risk estimation. 
Linlin Lindayani: An Exploration the Risk of Cardiovascular Disease in HIV-Positive Persons

\section{Introduction}

Cardiovascular disease is the leading cause of death not only in general population but also in patients with HIV (Human Immunodeficiency virus). Because of its high mortality rate, cardiovascular disease receives a major concern in the care and management of patients with HIV, especially those under antiretroviral therapy (ART). Previous research reported that there was a shift on the trend of caused of the death of patients with HIV. In the era before ART (pre-ART) was found only 5\% of deaths caused by heart disease, but now, in the era posts-ART, 32-42\% of HIV patients die due to heart disease (Krentz, Kliewer, \& Gill, 2015; Palella et al., 2016). Patients with HIV have 1.5 to 2 times the cardiovascular risk compared to people who are not infected with HIV and that increases with age (Chow et al., 2015; Triant, Lee, Hadigan, \& Grinspoon, 2007). Another study reported that about $58.3 \%$ of patients with HIV had at least one cardiovascular risk factor and $18.9 \%$ had two and more cardiovascular risk factors (Soliman et al., 2015). The risk of cardiovascular disease continues to increase in patients with HIV on ARTcabout $74.4 \%$ and $25.6 \%$ of naïve ART (Nsagha et al., 2015). In addition, the increased risk of heart disease in HIV patients is related to lifestyle and risk behavioral factors such as high rates of smoking, alcoholism, and drug use, and obesity (Grundy, 2016).

Heart rate variability (HRV) is a very sensitive tool for measuring the autonomic tone of the heart. It reflects beat-to-beat changes in RR intervals, which are related to ongoing interactions between two autonomic nervous system arms (Mittal, 2004). Heart rate changes can occur in response to mental or physical stress, heart or non-cardiovascular disease, or pharmacological or invasive treatment. Imbalance of the autonomic nervous system with a shift toward increased sympathetic and decreased vagal tone has been shown to be associated with a higher risk of cardiac death. Therefore HRV becomes an important and well-known tool in identifying patients who are at cardiovascular risk (Sakhuja, 2007). By recording heart activity through an electrocardiogram (EKG) for 5 minutes can detect a person's risk of heart disease. HRV is easy to do, convenient, noninvasive to patients, and also inexpensive (Mittal, 2004). It is able to detect the effects of external interference. Previous studies conducted in Denmark reported that there was a moderate autonomy disorder in HIV patients as measured by HRV (Askgaard, 2011; Wongcharoen, 2013). Several studies have shown that the statistical, geometrical, spectral and nonlinear analysis of HRV is a powerful tool for cardiovascular evaluation (Correia, 2006; Melillo, 2015).

In Indonesia there are still few studies that focus on early detection of cardiovascular disease in the general public (who are not infected with HIV) or HIV patients, whereas heart disease (a type of cardiovascular disease) is the first killer of Indonesian society. Thus, early detection and early prevention interventions for people at high risk in routine practice to reduce the incidence of cardiovascular disease are very necessary. So the purpose of this study was to identify the risk of cardivascular disease in patients with HIV using ECG short term.

\section{Method}

Study design

This study was used a descriptive comparative aims to identify CVD risk using ECG short term, namely HRV indicators in patients with HIV compared with healthy people

Population and Samples

The population in this study were patients with HIV and healthy people as controls in West Java. The inclusion criteria are diagnosed with HIV confirmed by medical record, age over 30 years, not diagnosed from heart disease or being treated for the heart disease. The inclusion criteria for healthy people were age over 30 years, not dignosed with cardivascular disease or receiving treatment of cardiovascular disease. Exclusion criteria are pregnant women, people with diabetes mellitus, and menopause. The sample technique that will be used is convinience sampling due to resource constraint. A total of 40 participant that consist of 20 patients with HIV and 20 healthy people were recruited 
Linlin Lindayani: An Exploration the Risk of Cardiovascular Disease in HIV-Positive Persons

in this study. Approval of ethical permission was obtained from the afiliated univeristy.

Measurement

Demographic data and medical history were collected using standard forms. Demographic data collected includes age and gender. Medical history including selfreported of years living with HIV and Under ART, and current CD4 counts.

Physical examination includes systolic and diastolic blood pressure, height, and weight. Blood pressure was measured by nurses through the brachial artery using a digital sphygmomanometer with an adult cuff on the day of data collection. The patient sits in an upright position in a quiet room; two consecutive blood pressure measurements were taken, and the average was recorded as the final result. Blood pressure is defined as high if systolic blood pressure $\geq 140 \mathrm{mmHg}$ and diastolic blood pressure $\geq 90 \mathrm{mmHg}$ (Pickering, 2005). Subjects were defined as thin if the BMI (body mass index) $\leq 18.5 \mathrm{~kg} /$ $\mathrm{m} 2$, normal (BMI ranging from 18.5 to 24.9 ), being overweight (BMI starting from 25.029.9), and obesity (BMI $\geq 30$ ).

The measurement of heart rate variability is carried out in a supine position in a quiet temperature-controlled room $(25-270 \mathrm{C})$, a 5-minute electrocardiograph (ECG) is recorded using lead II. The following five tests were carried out to assess autonomic function (Miller, 1987): 1) Stand active: Blood pressure is measured in the supine position and immediately after active standing. Blood pressure and heart rate response and the ratio of 30:15 (the ratio of the longest R-R interval of about 30 beats and the shortest interval of $\mathrm{R}-\mathrm{R}$ is calculated to be about 15 beats); 2). Deep breathing: expiration: inspiration ratio (E: I) and changes in heart rate measured during deep breathing; 3). Static grip test: Blood pressure response during isometric exercise is measured; 4). Cold pressor test: Blood pressure response to soak hands in ice cold water is assessed; 5). Cold face test: The response of blood pressure during the application of cold packs to the face is measured.

\section{Data analysis}

The Kolmogorov-Smirnov test of all variables was performed to test the normal distribution assumption. As the results of normality test found not normally distribute, therefore all the test was conducted using non parametric test. Comparisons between groups were performed using the Fisher exact test for binomial data ( $2 \times 2$ contingency tables) and the Mann-Whitney test for continuous data. Correlations were analyzed using non-parametric (Spearman) correlation and expressed by Spearman's rho. $\mathrm{P}<0.05$ was considered significant. P values between 0.05 and 0.1 are considered to be significant limits.

Table 1 Comparation of Demographic and Clinical Characteristics of Study Groups

\begin{tabular}{lccc}
\hline & $\begin{array}{c}\text { HIV group } \\
(\mathbf{n}=\mathbf{2 0})\end{array}$ & $\begin{array}{c}\text { Control group } \\
(\mathbf{n = 2 0})\end{array}$ & Significance \\
\hline Gender (\%) & $11(55)$ & $12(60)$ & 0.12 \\
Male & $9(45)$ & $8(40)$ & 0.43 \\
Female & $43(27-51)$ & $42(29-56)$ & 0.32 \\
Age (years) & $25(23-27)$ & $25(24-28)$ & 0.54 \\
Body Mass Index (kg/m2) & $76(69-81)$ & $74(67-89)$ & 0.67 \\
Diastolic blood pressure (mmHg) & $123(116-134)$ & $125(115-139)$ & - \\
Systolic blood pressure (mmHg) & $9.1(5.8-14.3)$ & - & - \\
Duration of HIV (years) & $7.3(3.6-8.1)$ & - & - \\
Duration of ART (years) & $432(342-676)$ & - & \\
CD4 cell cound (cell/mm3) & & \\
\hline
\end{tabular}

Note: ns: non significance. 
Linlin Lindayani: An Exploration the Risk of Cardiovascular Disease in HIV-Positive Persons

Table 2 Heart Rate Variability Based On Time Domain Analysis

\begin{tabular}{lccc}
\hline & $\begin{array}{c}\text { HIV group } \\
(\mathbf{n}=\mathbf{2 0})\end{array}$ & $\begin{array}{c}\text { Control group } \\
(\mathbf{n = 2 0})\end{array}$ & Significance \\
\hline Mean NN (ms) & $902(845-987)$ & $978(891-1,113)$ & $\mathrm{P}<0.05$ \\
SDNN (ms) & $45(37-67)$ & $51(43-76)$ & 0.13 \\
RMSSD (ms) & $29(20-48)$ & $26(22-40)$ & 0.47 \\
pNN50 (\%) & $5(1-20)$ & $4(1-8)$ & 0.32 \\
Note: Values are median (25 percentile-75 percentile). &
\end{tabular}

\section{Results}

A total of 20 patients with HIV and 20 healthy people. The majority of people with HIV were male and aged range between 27 to 51 years old. While, in control group, majority were male and aged ranged 29 to 56 years old. The mean duration of living with HIV was 9 years (range 5.8-14.3). There were no significant different between HIV group and control group (healthy people) in tearm of age, gender, body mass index, systolic and diastolic blood pressure (Table 1).

The results of heart rate variability based on time domain analysis is described in Table 2. The means mean normal-to-normal (NN) was significantly lower in HIV patients compared to controls (978 vs vs $902 \mathrm{~ms} ; \mathrm{p}<0.05$ ). No differences were found between groups regarding Standard deviation of NN (SDNN), Square root of the mean squared difference of successive NN-intervals (RMSSD) and Percent of differences between adjacent NN intervals greater than $50 \mathrm{~ms}$ (pNN50).

Mean NN: mean normal-to-normal, SDNN: Standard deviation of NN; RMSSD:

Square root of the mean squared difference of successive NN-intervals; pNN50:

Percent of differences between adjacent $\mathrm{NN}$ intervals greater than $50 \mathrm{~ms}$.

\section{Discussion}

Our study presence of autonomic dysfunction in a group of HIV compared to the healthy group. Decrease in mean NN indicating a decrease mainly in parasympathetic tone (Askgaard, 2011). Some evidence has reported that there is an increased risk of cardiovascular disease in HIV patients (Freiberg et al., 2013). A recent analysis of HIV patients aged 30-50 years using bilateral intima-media (cIMT) found that $19.2 \%$ of HIV-infected patients had an increased risk of cardiovascular disease (Mosepele et al., 2017). A study from the Netherlands reported that around $33 \%$ of HIV patients measured by FRS had a high risk of cardiovascular disease (Krikke et al., 2016). This was supported by a cross-sectional study conducted in Croatia $(n=254)$ reporting that people with HIV had a high risk of CVD, ranging from $27.2 \%$ measured by FRS to $51.6 \%$ for DAD scores (Begovac et al., 2015). A study from Zimbabwe in HIV-positive people $(\mathrm{n}=215$, mean age $=39.8-42.0$, under ART $3.9(\mathrm{SD}=$ 3.4)) also reported $1.4 \%$ prevalence of high risk cardivascular disease in the next ten years (Zhou et al., 2015).

The underlying mechanism by which HIV driving excess CVD risk is not clear but likely involves a combination of factors including the virus itself, side effects of cART and the burden of traditional risk factors. HIV infection is associated with an increased risk of CVD surrogate marker, such as carotid intima-media thickness, arterial stiffness, endothelial dysfunction (Obel et al., 2007; Oliviero et al., 2009; van Vonderen et al., 2009). In addition, cART increased risk of CVD by elevating LDL, total cholesterol, triglyceride, and HDL (Kiage et al., 2013; Nsagha et al., 2015). Moreover, the increasing vulnerability of CVD depending on lifestyle and risk behaviors factors such as smoking, alcoholism, and illicit drug use, as well obesity (Grundy, 2016).

Heart rate variability (HRV) is a very sensitive tool for measuring the heart's autonomic tone. Previous studies conducted in Denmark reported that there was moderate autonomic disorder in HIV patients measured using HRV (Askgaard, 2011 \& Wongcharoen, 2013). Previous study shown that statistical, geometric, spectral and nonlinear analyzes of 
Linlin Lindayani: An Exploration the Risk of Cardiovascular Disease in HIV-Positive Persons

HRV are powerful tools for cardiovascular evaluation (Binici, 2011). Heart rate variability (HRV) reflects beat-to-beat changes in RR intervals, which are related to ongoing interactions between two autonomic nervous system arms (Mittal, 2004). The sinus node, the main pacemaker, presents its own intrinsic activity; however, various internal and external stimuli that change the balance between sympathetic and vagal tone affect the final base heart rate. Heart rate changes can occur in response to mental or physical stress, heart or non-cardiovascular disease, or pharmacological or invasive treatment. Imbalance of the autonomic nervous system with a shift toward increased sympathetic and decreased vagal tone has been shown to be associated with a higher risk of cardiac death. Therefore HRV becomes an important and well-known tool in identifying patients who are at cardiovascular risk (Sakhuja, 2007). HRV measurement becomes an important tool in detecting damage and predicting autonomic prognosis in several neurological disorders as well. Heart rate (HR) has been extensively studied in cardiac patients, especially in patients with acute myocardial infarction (AMI) and also in patients with congestive heart failure (CHF) or left ventricular dysfunction (LV). The majority of studies have shown that patients with reduced or abnormal HRV have an increased risk of death within a few years after AMI or after the diagnosis of CHF / LV dysfunction. Various methods used for HRV measurement such as time domain, spectral, and non-linear have been used in risk stratification.

\section{Conclusion}

Our study found a presence of autonomic dysfunction as presented in heart rate variability analysis in patients with HIV indicating decreased in the parasympatic tone of the heart. Althought others parameter in heart rate variability were not significantly different between HIV group and healthy group. Eearly identification of the risk of CVD is important and may inform the implementation of preventive measure by identification of high-risk people who may be candidate for intervention.
The authors declare that they have no competing interests.

This study was funded by the Ministry of Research, Technology, and Higher Education, Indonesia.

\section{References}

Askgaard, G., et al. (2011). Decreased heart rate variability in HIV positive patients receiving antiretroviral therapy: Importance of blood glucose and cholesterol. PLoS One, 6(5), p. e20196.

Begovac, J., Dragović, G., Višković, K., Kušić, J., Perović Mihanović, M., Lukas, D., \& Jevtović, Đ. (2015). Comparison of four international cardiovascular disease prediction models and the prevalence of eligibility for lipid lowering therapy in HIV infected patients on antiretroviral therapy. Croatian Medical Journal, 56(1), p.14-23. doi:10.3325/cmj.2015.56.14.

Binici, Z., Mouridsen, M.R., Kober, L., \& Sajadieh, A. (2011). Decreased nighttime heart rate variability is associated with increased stroke risk. Stroke, 42(11), 31963201. doi:10.1161/strokeaha.110.607697.

Chow, D., et al. (2015). HIV and coronary artery calcium score: Comparison of the Hawaii Aging with HIV Cardiovascular Study and Multi-Ethnic Study of Atherosclerosis (MESA) cohorts. HIV Clin Trials, 16(4), p. $130-8$.

Correia, D. (2006). Power spectral analysis of heart rate variability in HIV-infected and AIDS patients. Pacing Clinical Electrophysiology, 29(1), p. 53-8.

Freiberg, M.S., \& K. So-Armah. (2016). HIV and cardiovascular disease: We need a mechanism, and we need a plan. J Am Heart Assoc, 5(3).

Grundy, S.M. (2016). Metabolic syndrome update. Trends Cardiovasc Med, 26(4), 364373. doi:10.1016/j.tcm.2015.10.004.

Melillo, P. (2015). Automatic prediction of 
Linlin Lindayani: An Exploration the Risk of Cardiovascular Disease in HIV-Positive Persons

cardiovascular and cerebrovascular events using heart rate variability analysis. PLoS One, 10(3), e0118504

Mosepele, M., et al. (2017). Cardiovascular disease risk prediction by the American College of Cardiology (ACC)/American Heart Association (AHA) Atherosclerotic Cardiovascular Disease (ASCVD) risk score among HIV-infected patients in sub-Saharan Africa. PLoS One, 12(2), p. e0172897.

Nsagha, D.S., et al. (2015). Risk factors of cardiovascular diseases in HIV/AIDS patients on HAART. Open AIDS J, 9, p. 51-9.

Kiage, J.N., et al. (2013). Cardiometabolic risk factors among HIV patients on antiretroviral therapy. Lipids Health Dis, 12, p. 50.

Krentz, H.B., G. Kliewer, \& M.J. Gill. (2005). Changing mortality rates and causes of death for HIV-infected individuals living in Southern Alberta, Canada from 1984 to 2003. HIV Med, 6(2), p. 99-106.

Krikke, M., et al. (2016). Cardiovascular risk prediction in HIV-infected patients: Comparing the Framingham, Atherosclerotic Cardiovascular Disease Risk Score(ASCVD), Systematic Coronary Risk Evaluation for the Netherlands (SCORE-NL) and Data Collection on Adverse Events of Anti-HIV Drugs (D:A:D) risk prediction models. HIV Med, 17(4), p. 289-97.

Obel, N., et al. (2007). Ischemic heart disease in HIV-infected and HIV-uninfected individuals: A population-based cohort study. Clin Infect Dis, 44(12), p. 1625-31.

Oliviero, U., et al. (2009). Human immunodeficiency virus per se exerts atherogenic effects. Atherosclerosis, 204(2), p. 586-9.

Palella, F.J., Jr., et al. (2006). Mortality in the highly active antiretroviral therapy era: Changing causes of death and disease in the
HIV outpatient study. J Acquir Immune Defic Syndr, 43(1), p. 27-34.

Pickering, T.G. (2005). Do we really need a new definition of hypertension? Journal of Clinical Hypertension, 7(12), p. 702-4.

Sakhuja, A. (2007). Heart rate variability and autonomic function tests in HIV positive individuals in India. Clinical Autonomy Research, 17(3), p. 193-6.

Soliman, E.Z., et al. (2015). Baseline cardiovascular risk in the INSIGHT strategic timing of antiretroviral treatment trial. $H I V$ Medicine, 16(0 0), p. 46-54.

Triant, V.A., et al. (2007). Increased acute myocardial infarction rates and cardiovascular risk factors among patients with human immunodeficiency virus disease. The Journal of Clinical Endocrinology and Metabolism, 92(7), p. 2506-2512.

van Vonderen, M.G., et al. (2009). Carotid intima-media thickness and arterial stiffness in HIV-infected patients: The role of HIV, antiretroviral therapy, and lipodystrophy. $J$ Acquir Immune Defic Syndr, 50(2), p. 15361.

Wongcharoen, W., et al. (2013). Heart rate variability and heart rate turbulence in HIVinfected patients receiving combination antiretroviral therapy. Ann Noninvasive Electrocardiol, 18(5), p. 450-6.

Yeragani, V.K., Pohl, R., Berger, R. (1994). Relationship between age and heart rate variability in supine and standing postures: A study of spectral analysis of heart rate. Pediatric Cardiology, 15, 14-20. doi:10.1007/ BF00797000.

Zhou, D.T., et al. (2015). Dyslipidemia and cardiovascular disease risk profiles of patients attending an HIV treatment clinic in Harare, Zimbabwe. HIV AIDS, 7, p. 145-55. 